\title{
Análise clínica do diâmetro do forame dos molares de acordo com a faixa etária e diagnóstico pulpar e perirradicular
}

\author{
Clinical analysis of the molar's foramen diameter according to the age range and pulp and \\ periradicular diagnosis \\ Análisis clínico del diámetro del foramen molar según rango de edad y diagnóstico pulpar y \\ perirradicular
}

Recebido: 24/04/2021 | Revisado: 03/05/2021 | Aceito: 06/05/2021 | Publicado: 21/05/2021

Giovana Domitila Rafagnin

ORCID: https://orcid.org/0000-0002-5401-2301

Faculdade São Leopoldo Mandic, Brazil

E-mail: giovanarafagnin@hotmail.com

Augusto Shoji Kato

ORCID: https://orcid.org/0000-0003-2971-0906

Faculdade de Odontologia de Bauru, Brazil

E-mail: endo.kato@gmail.com

Ana Grasiela da Silva Limoeiro

ORCID: https://orcid.org/0000-0003-4633-720X

Faculdade de Ilhéus, Brazil

E-mail: grasielalimoeiro@gmail.com

Rina Andrea Pelegrine

ORCID: https://orcid.org/0000-0003-4175-2121

Faculdade São Leopoldo Mandic, Brazil

E-mail: rinapelegrine@terra.com.br

Laura De Vito

ORCID: https://orcid.org/0000-0003-3446-8966 Faculdade São Leopoldo Mandic, Brazil E-mail: laura@gmail.com

Carlos Eduardo Fontana

ORCID: https://orcid.org/0000-0002-2868-6839 Pontifícia Universidade Católica, Brazil

E-mail: ceffontana@hotmail.com

Daniel Guimarães Pedro Rocha

ORCID: https://orcid.org/0000-0001-9792-2260 Pontifícia Universidade Católica, Brazil E-mail:dnlrocha@uol.com.br

Ricardo Machado

ORCID: https://orcid.org/0000-0002-1901-7270

Universidade de Blumenau, Brazil

E-mail: ricardo.machado.endo@gmail.com

Carlos Eduardo Da Silveira Bueno

ORCID: https://orcid.org/0000-0002-2675-0884

Faculdade São Leopoldo Mandic, Brazil

E-mail: carlosesbueno@terra.com.br

\begin{abstract}
Resumo
Introdução: A compreensão das características do diâmetro foraminal contribui para o trabalho do clínico pois permite a seleção dos instrumentos apropriados para o preparo dos canais radiculares. O objetivo deste trabalho foi avaliar o diâmetro foraminal, in vivo, em canais de molares superiores e inferiores e verificar a influência de fatores como faixa etária e diagnóstico pulpar e perirradicular. Métodos: 150 canais radiculares de 48 primeiros e segundos molares superiores e inferiores, foram divididos em grupos de acordo com a faixa etária: A1 - Menores de 15 anos; A2 - 16 a 34 anos e A3 - 34 a 50 anos, e de acordo com o diagnóstico: Pulpite irreversível; necrose pulpar e necrose pulpar com periodontite apical assintomática. Limas tipo K foram inseridas até o comprimento real do dente (CRD) para a seleção do instrumento anatômico foraminal (IAF). O diâmetro inicial da ponta (D0) do IAF foi considerado para a determinação do diâmetro foraminal. Os dados foram analisados por meio da análise de variância a três critérios e para as comparações múltiplas empregou-se o teste de Tukey. Resultados: Houve diferença estatística significante na interação entre faixa etária e diagnóstico ( $\mathrm{p}<0.001)$, sendo que dentes de pacientes mais jovens (A1) com pulpite irreversível apresentaram maiores diâmetros. Ainda, verificou-se que, independente da faixa etária e do diagnóstico, o diâmetro foi significativamente influenciado pelo tipo de dente/canal ( $\mathrm{p}<0.001)$. Conclusão: O diâmetro foraminal
\end{abstract}


pode variar conforme o tipo de dente/canal radicular, sendo que faixa etária e diagnóstico também influenciam os resultados quando analisados concomitantemente.

Palavras-chave: Anatomia; Ápice dentário; Endodontia.

\begin{abstract}
Introduction: The understanding of the characteristics of the foraminal diameter contributes to the work of the clinician because it allows a selection of the appropriate instruments for the root canals preparation. The aim of this study was to evaluate the foraminal diameter, in vivo, in upper and lower molar canals and to verify an influence of factors such as age group and pulp and periradicular diagnosis. Methods: 150 root canals of 48 upper and lower first and second molars, were divided into groups according to age group: A1 - Under 15 years old; A2 - 16 to 34 years old and A3 - 34 to 50 years old, and according to the diagnosis: Irreversible pulpitis; pulp necrosis and pulp necrosis with asymptomatic apical periodontitis. K files were inserted up to the actual tooth length for a foraminal anatomical instrument selection (FAI). The initial diameter of the tip (D0) of the FAI was considered for the determination of foraminal diameter. The data were analyzed using analysis of variance with three criteria and for multiple comparisons the Tukey test was used. Results: There was a statistically significant difference in the interaction between age group and diagnosis $(\mathrm{p}<0.001)$, with the teeth of younger patients (A1) with irreversible pulpitis having larger diameters. Furthermore, it was found that, regardless of age and diagnosis, the diameter was significantly influenced by the type of tooth / canal ( $\mathrm{p}<0.001)$. Conclusion: The foraminal diameter can vary according to the type of tooth / root canal, with age and diagnosis also influencing the results when analyzed concomitantly.
\end{abstract}

Keywords: Anatomy; Dental apex; Endodontics.

\title{
Resumen
}

Introducción: La comprensión de las características del diámetro foraminal contribuye al trabajo del clínico porque permite seleccionar los instrumentos adecuados para la preparación de los conductos radiculares. El objetivo de este estudio fue evaluar el diámetro foraminal, in vivo, en los conductos molares superiores e inferiores y verificar la influencia de factores como el grupo de edad y el diagnóstico pulpar y perirradicular. Métodos: 150 conductos radiculares de 48 primeros y segundos molares superiores e inferiores, fueron divididos en grupos según grupo de edad: A1 - Menores de 15 años; A2 - 16 a 34 años y A3 - 34 a 50 años, y según diagnóstico: Pulpitis irreversible; necrosis pulpar y necrosis pulpar con periodontitis apical asintomática. Las limas de tipo K se insertaron hasta la longitud real del diente (CRD) para una selección de instrumentos anatómicos foraminales (IAF). Se consideró el diámetro inicial de la punta (D0) del IAF para la determinación del diámetro foraminal. Los datos se analizaron mediante análisis de varianza con tres criterios y para comparaciones múltiples se utilizó la prueba de Tukey. Resultados: Hubo una diferencia estadísticamente significativa en la interacción entre el grupo de edad y el diagnóstico (p <0,001), y los dientes de los pacientes más jóvenes (A1) con pulpitis irreversible tenían diámetros más grandes. Además, se encontró que, independientemente de la edad y el diagnóstico, el diámetro fue influenciado significativamente por el tipo de diente / canal ( $\mathrm{p}<0.001)$. Conclusión: El diámetro foraminal puede variar según el tipo de diente / conducto radicular, y la edad y el diagnóstico también influyen en los resultados cuando se analizan concomitantemente.

Palabras clave: Anatomía; Ápice dental; Endodoncia.

\section{Introdução}

A terapia endodôntica visa a limpeza do sistema de canais radiculares (SCR) através da ação física dos instrumentos endodônticos nas paredes do canal, associada a ação química das soluções irrigadoras, sendo estes componentes essenciais para o sucesso da endodontia (Lopes et al., 2015).

A compreensão da anatomia do SCR, bem como das características do terço apical, principalmente do diâmetro foraminal, contribui para o trabalho do clínico, especificamente nos procedimentos de limpeza e conformação do terço apical (Abarca et al., 2014; Jou et al., 2004; Peters \& Peters, 2011) permitindo a seleção dos instrumentos apropriados para a instrumentação, evitando desgastes desnecessários de dentina ou a permanência de áreas não instrumentadas, e também facilita a escolha da lima adequada para a realização da patência foraminal, visto que, segundo Schilder (1974), esta etapa pode ser realizada com a utilização de um instrumento compatível com o diâmetro do forame, passando por toda a extensão radicular e ultrapassando o limite de instrumentação, sem causar alterações na sua forma original, permitindo a manutenção da permeabilidade do canal.

Uma maneira clínica para determinar o diâmetro foraminal de forma individualizada para cada canal radicular, é através da escolha do instrumento anatômico foraminal (IAF), que se caracteriza por ser o primeiro instrumento que se 
adapta de forma mais justa no comprimento real do dente, considerando o diâmetro inicial da sua ponta (D0). A partir dele, realiza-se o planejamento para a escolha das limas a serem utilizadas no preparo mecânico.

De acordo com estudos de anatomia interna, os canais radiculares dos molares superiores e inferiores apresentam características distintas entre si em relação a secção transversal (forma e diâmetro médio dos canais), tanto da região cervical quanto da apical (Demiriz et al., 2018; Madeira \& Rizzolo, 2007; Versiani et al. 2015; Wolf et al., 2017).

Levando-se em consideração o conhecimento da anatomia foraminal provenientes de pesquisas ex vivo (Campelo et al., 2019; Levorato et al. 2011; Vier et al., 2004; Wu et al., 2000; Wu et al., 2002) este trabalho teve por objetivo obter o diâmetro do forame apical das raízes de primeiros e segundos molares superiores e inferiores, in vivo, visando identificar se fatores como faixa etária dos pacientes e os diagnósticos pulpar e perirradicular influenciam estes resultados.

\section{Materiais e Métodos}

Os participantes foram orientados quanto aos procedimentos éticos e de pesquisa, e este estudo clínico foi aprovado pelo comitê de ética em pesquisa local, sob o número de parecer 3.324.472. Um total de 150 canais radiculares de 48 primeiros e segundos molares superiores e inferiores de 45 pacientes entre 9 e 50 anos, encaminhados para tratamento endodôntico no Centro de Especialidades Odontológicas na cidade de Francisco Beltrão, Paraná, Brasil, durante os meses de Setembro de 2018 à Fevereiro de 2019, foram selecionados para o estudo com base em um cálculo amostral, considerando a existência de faixa etária e diagnóstico como variáveis independentes, sendo adotado o modelo de análise de variância e utilizado o programa G*Power 3. 1.5 (Heinrich Heine, Universita Dusseldorf) com o nível de significância de 5\% e poder de 80\%.

Os grupos foram divididos de acordo com a faixa etária, considerando estudos anteriores (Gani \& Visvisian, 1999; Vier et al., 2004) em: A1 - Menores de 15 anos (n=14); A2 - 16 a 34 anos (n=14) e A3 - 34 a 50 anos (n=14). E, de acordo com o diagnóstico pulpar e perirradicular em: Pulpite irreversível $(n=36)$; necrose pulpar $(n=36)$ e necrose pulpar com periodontite apical assintomática $(\mathrm{n}=36)$.

Para a classificação quanto ao tipo de diagnóstico, levou-se em consideração a resposta do teste de sensibilidade pulpar realizado com gás refrigerante (Endo-ice, Maquira Indústria de Produtos Odontológicos S.A., Maringá, Brasil) e a avaliação radiográfica a partir de uma radiografia periapical realizada previamente e digitalizada. Dentes com comprometimento pulpar e que responderam positivo ao teste de sensibilidade pulpar foram incluídos no grupo de pulpite irreversível; dentes que não responderam ao teste e que não apresentaram alteração radiográfica apical foram diagnosticados como necrose pulpar; e aqueles que não responderam ao teste e radiograficamente apresentaram área de rarefação óssea apical foram classificados como necrose pulpar com periodontite apical assintomática. Foram excluídos do estudo dentes que apresentaram rizogênese incompleta, reabsorção radicular externa apical visível radiograficamente, raízes com ângulo de curvatura acima de $30^{\circ}$ pelo método de Schneider, canais visivelmente atrésicos, canais onde a patência não pôde ser obtida, dentes com pulpite reversível e dentes que já apresentavam tratamento endodôntico primário.

Diante da definição do diagnóstico pulpar e perirradicular, um examinador único realizou o acesso a câmara pulpar com pontas diamantadas 1014 (KG SORENSEN, Cotia, Brasil), seguido pela patência, estabelecida através de uma lima K\#10 (Dentsply Maillefer, Ballaigues, Suiça). O preparo cervical foi realizado com brocas Gates-Glidden (Dentsply Maillefer) em ordem crescente 1, 2 e 3, e o comprimento real do dente (CRD) determinado eletronicamente com localizador foraminal (Finepex, Schuster, Santa Maria, Brasil), utilizando lima tipo K\#15.

Limas manuais (Dentsply Maillefer) de primeira e segunda série, de taper 0,02, tiveram seus cabos vedados por uma fita, a fim de dificultar a identificação visual pelo operador, e foram sucessivamente introduzidas com movimento de cateterismo nos canais radiculares até identificar a que melhor se adaptasse no CRD. O diâmetro dessa lima foi registrado, considerando o D0 do instrumento. 
Para a realização da análise estatística, a dimensão registrada no banco de dados informatizado foi representada pelo maior instrumento capaz de atingir o CRD, estabelecido eletronicamente. Os efeitos da faixa etária, do tipo de dente e seus canais e do diagnóstico, bem como suas interações, no diâmetro D0 dos instrumentos utilizados no qual se baseou a determinação do diâmetro foraminal foram investigados por meio da análise de variância a três critérios. Para as comparações múltiplas, empregou-se o teste de Tukey. Os cálculos estatísticos foram conduzidos no programa SPSS 23 (SPSS Inc., Chicago, IL, EUA), adotando-se o nível de significância de $5 \%$.

\section{Resultados}

De acordo com análise de variância a três critérios, verificou-se que não houve influência da interação entre a faixa etária, o tipo de dente/canal e o diagnóstico $(\mathrm{p}=0.967)$. As interações duplas entre faixa etária e dente/canal $(\mathrm{p}=0.673)$ bem como entre diagnóstico e dente/canal $(\mathrm{p}=0.086)$ não se mostraram estatisticamente significativas. Entretanto, a interação entre faixa etária e o diagnóstico revelou-se estatisticamente significativa ( $\mathrm{p}<0.001)$, conforme expresso na Tabela 1.

Tabela 1 - Valores médios e desvio padrão do diâmetro foraminal (mm), segundo a faixa etária e o diagnóstico pulpar e perirradicular, independentemente dos tipos de dente molar e canal.

Idade

\section{Diagnóstico}

\begin{tabular}{l|l|l|l}
\hline & \multicolumn{1}{|c|}{ Pulpite Irreversível } & \multicolumn{1}{|c}{ Necrose Pulpar } & \multicolumn{1}{c}{ Periodontite Apical } \\
\hline A1 - até 15 anos & $0,38(0,09) \mathrm{Aa}$ & $0,25(0,06) \mathrm{Cb}$ & $0,33(0,09) \mathrm{Ba}$ \\
\hline A2 $-\mathbf{1 6}$ a 34 anos & $0,30(0,08) \mathrm{Ab}$ & $0,29(0,06) \mathrm{Aa}$ & $0,26(0,10) \mathrm{Bb}$ \\
\hline $\mathbf{A 3}-\mathbf{3 5}$ a 50 anos & $0,28(0,08) \mathrm{Ab}$ & $0,26(0,09) \mathrm{Ab}$ & $0,27(0,11) \mathrm{Ab}$ \\
\hline
\end{tabular}

Legenda: Médias seguidas por letras maiúsculas distintas indicam diferença estatisticamente significativa no diâmetro diante dos diagnósticos, considerando-se separadamente cada faixa etária. Médias seguidas por letras minúsculas distintas indicam diferença estatisticamente significativa entre faixas etárias, considerando-se separadamente cada diagnóstico.

Fonte: Autores.

O diagrama de colunas abaixo (Figura 1), demonstra os valores médios do instrumento anatômico foraminal de acordo com a faixa etária e o diagnóstico pulpar e perirradicular, independente do dente ou do canal radicular avaliados.

Figura 1 - Diâmetros correspondentes ao instrumento anatômico foraminal segundo a faixa etária e o diagnóstico pulpar e perirradicular.

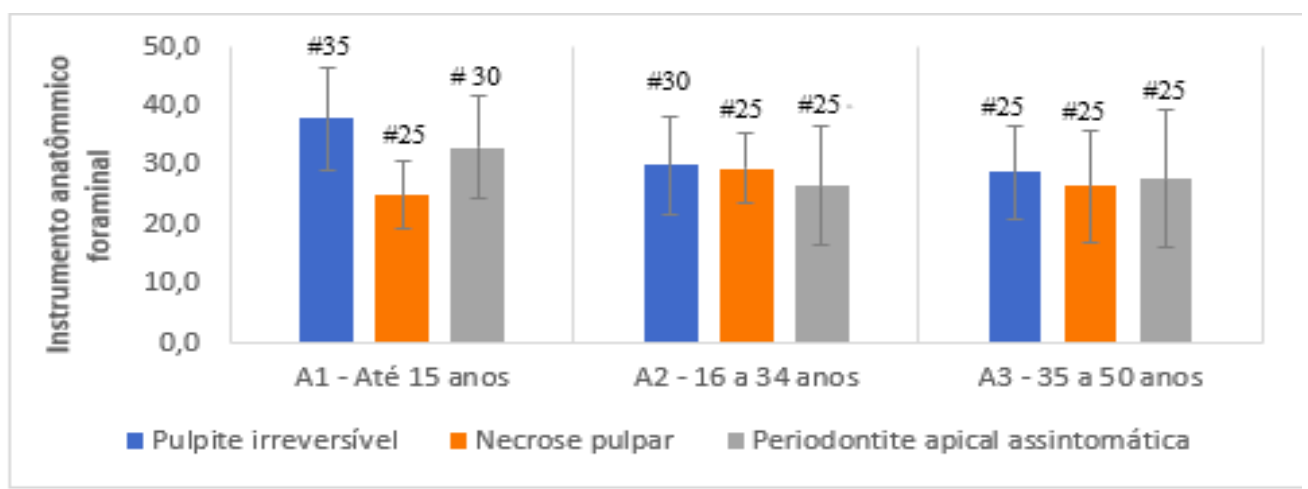


A análise de variância a três critérios também revelou que, independente da faixa etária e do diagnóstico, o diâmetro foraminal foi significativamente influenciado pelo tipo de dente/canal $(\mathrm{P}<0.001)$ (tabela 2 ), sendo que pelo teste de Tukey, observou-se que os maiores diâmetros foram encontrados nos canais distal de segundos molares inferiores $(0,47 \mathrm{~mm})$ e palatino de primeiros molares superiores $(0,40 \mathrm{~mm})$.

Tabela 2 - Valores médios e desvio padrão do diâmetro foraminal (mm) de acordo com o tipo de dente molar e seus canais radiculares, independentemente da faixa etária e do diagnóstico pulpar e perirradicular.

\begin{tabular}{l|l|l}
\multicolumn{1}{c}{ Dente } & \multicolumn{1}{c}{ Canal } & \multicolumn{1}{c}{ Diâmetro } \\
\hline \multirow{3}{*}{ Primeiro molar superior } & Mésio-vestibular & $0,25(0,07) \mathrm{D}$ \\
& Mésio-vestibular (MV2) & $0,19(0,05) \mathrm{D}$ \\
& Disto-vestibular & $0,27(0,06) \mathrm{CD}$ \\
& Palatino & $0,40(0,08) \mathrm{AB}$ \\
\hline \multirow{3}{*}{ Segundo molar superior } & Mésio-vestibular & $0,22(0,03) \mathrm{D}$ \\
& Disto-vestibular & $0,21(0,06) \mathrm{D}$ \\
& Palatino & $0,38(0,08) \mathrm{B}$ \\
\hline \multirow{3}{*}{ Primeiro molar inferior } & Mésio-vestibular & $0,25(0,06) \mathrm{D}$ \\
& Mésio-lingual & $0,25(0,07) \mathrm{D}$ \\
& Disto-vestibular & $0,35(0,08) \mathrm{BC}$ \\
& Disto-lingual & $0,37(0,08) \mathrm{B}$ \\
& Distal & $0,36(0,10) \mathrm{B}$ \\
\hline \multirow{3}{*}{ Segundo molar inferior } & Mésio-vestibular & $0,25(0,05) \mathrm{D}$ \\
& Mésio-lingual & $0,27(0,06) \mathrm{CD}$ \\
\hline
\end{tabular}

Legenda: Médias seguidas por letras maiúsculas distintas indicam diferença estatisticamente significativa entre dentes/respectivos canais quanto ao diâmetro. Fonte: Autores.

\section{Discussão}

A compreensão das dimensões da anatomia interna dos dentes tem sido um desafio desde os estudos de Kerekes \& Tronstad (1977) e Kuttler (1955). A estimativa do diâmetro do forame apical in vivo e de forma individualizada para cada canal radicular, torna-se importante pois permite a seleção dos instrumentos adequados para a completa instrumentação dos canais radiculares.

De acordo com Wu et al (2002), o diâmetro da lima que melhor se adapta ao terço apical do canal principal é sempre menor do que o tamanho do real do forame, ou seja, a determinação do diâmetro foraminal através de instrumentos endodônticos não é totalmente precisa. Entretanto, segundo Contreras, Zinman e Kaplan (2001), Pecora et al (2005) e Tan e Messer (2002) esta situação pode ser minimizada mediante a realização do preparo cervical previamente à escolha do IAF, etapa executada em todos os canais radiculares deste estudo, permitindo uma avaliação mais próxima da realidade. Dessa maneira, os resultados obtidos foram muito semelhantes aos apresentados em trabalhos ex vivo, de análise microscópica do diâmetro apical (Morfis et al., 1994; Pecora et al., 2005; Wu et al., 2000), e superiores aos encontrados em trabalho in vivo anterior (Kfir et al., 2006).

Dentes jovens costumam apresentar câmara pulpar e canais radiculares mais amplos quando comparados a pacientes de maior faixa etária, que exibem câmara pulpar e canais radiculares mais atrésicos, sendo a idade um fator modificador da anatomia dental. Entretanto, a interação entre faixa etária e tipo de dente ou canal neste estudo, não mostrou diferença estatística significante em relação ao diâmetro foraminal, corroborando com estudo anterior (Vier et al., 2004). Porém, essa diferença foi verificada na análise simultânea de idade e diagnóstico pulpar e perirradicular.

Acredita-se, então, em um impacto combinado entre as características clínicas do dente, diagnóstico e função dos tecidos dentais, somados ao envelhecimento (Nitzan et al., 1986). Ou seja, a morfologia dental é influenciada pelo tempo, devido a aposição contínua de dentina secundária e de cemento, e, pelas agressões do meio em que a polpa é exposta, como por exemplo, hábitos 
parafuncionais, trauma dental, processos cariosos ou restauradores extensos, estimulando a formação de dentina terciária e também de cemento (Singh et al., 2013). Para Kuttler (1955), a espessura do cemento a ser formada depende mais da força mastigatória a que este dente é submetido do que o tempo em que este dente se encontra em função propriamente dita.

Independente do dente/canal radicular avaliado, os pacientes mais jovens (A1) apresentaram os maiores valores do diâmetro foraminal para o diagnóstico de pulpite irreversível. Nesse contexto, acredita-se que, por se tratar de um dente jovem com uma condição patológica sintomática, permaneceu exposto às agressões do meio bucal por um período de tempo menor, visto que o atendimento para estes pacientes costuma ser imediato. Consequentemente, as características anatômicas de canal radicular amplo estarão mantidas.

Verificou-se ainda que, no grupo A1, dentes com periodontite apical assintomática apresentaram maiores valores para o diâmetro foraminal quando comparados aos canais de dentes com diagnóstico de necrose dentro da mesma faixa etária. De acordo com Ricucci et al., (2019), dentes com alterações apicais costumam apresentar reabsorção de cemento e dentina, mesmo que ainda não seja visível radiograficamente. Esse processo reabsortivo ocorre de forma variada e leva a perda da constrição apical e ao aumento da região foraminal (Ricucci et al., 2019). Por outro lado, no grupo A2, o diâmetro foraminal dos canais com periodontite apical assintomática foi significativamente menor se comparado aos outros dois diagnósticos, e no grupo A3 (35 a 50 anos) nenhuma diferença significativa entre os diâmetros foraminais dos canais com qualquer um dos diagnósticos analisados foi encontrada. Dessa forma, pode-se constatar que não somente o diagnóstico pulpar e perirradicular influencia as características do diâmetro foraminal, mas que a faixa etária do paciente e possivelmente as agressões do meio em que esse dente foi exposto (abrasão, atrição, processos restauradores profundos, cárie, doença periodontal), também devem ser levados em consideração.

Dados como presença e extensão de restaurações, hábitos parafuncionais e histórico de trauma, não foram considerados para esta pesquisa, o que talvez possa explicar a falta de homogeneidade entre os resultados encontrados na tabela 1 . Ao se analisar apenas o tipo de dente e canal, sem considerar a faixa etária e o diagnóstico, houve diferença estatística significante entre eles $(\mathrm{p}<0.001)$. Os maiores diâmetros foraminais encontrados foram nos canais distal de segundos molares inferiores $(0,47 \mathrm{~mm})$, e palatino de primeiros molares superiores $(0,40 \mathrm{~mm})$, corroborando com estudo ex vivo de Green (1958) e Morfis (1994). Os dados encontrados são, de certa forma, previsíveis devido as características anatômicas particulares destas raízes que são mais amplas, considerando que a anatomia interna segue, em linhas gerais, a externa.

O diâmetro médio foraminal do canal distal dos segundos molares inferiores foi de $0,47 \mathrm{~mm}$ e dos primeiros molares inferiores (disto-vestibular, disto-lingual ou distal único) variou entre 0,35mm e 0,37 mm. Dessa forma, o instrumento anatômico foraminal para canais distais de molares inferiores poderia ser determinado entre \#35 e \#45, semelhante aos resultados encontrados em trabalhos ex vivo anteriores (Green, 1958; Morfis et al., 1994; Wu et al. 2000) sobre anatomia apical, e alguns valores levemente superiores em comparação com outros trabalhos (Abarca et al., 2014; Marroquin et al., 2004).

Em relação ao diâmetro foraminal do canal palatino de primeiros e segundos molares superiores, o instrumento anatômico foraminal oscilaria entre \#35 e \#40, corroborando com trabalhos de Green (1958), muito próximo aos resultados de Abarca et al., (2014) e Marroquin et al., (2004), porém diâmetros superiores aos trabalhos de e Levorato et al. (2011), Morfis et al., (1994) Wu et al., (2000).

De uma maneira geral, a média do diâmetro foraminal para os canais mesiais, tanto de molares superiores quanto inferiores, bem como canal disto-vestibular dos molares superiores foi de 0,24 mm, alternando entre um instrumento anatômico \#20 e \#25, novamente apresentando resultados muito semelhantes aos encontrados em trabalhos ex vivo anteriores (Abarca et al., 2014; Marroquin et al., 2004).

Frente a diversidade de sistemas mecanizados, de diferentes calibres e diâmetros, disponíveis para o preparo mecânico dos canais radiculares, a determinação clínica e de forma individualizada do diâmetro foraminal, permite ao clínico planejar e 
selecionar a lima indicada para a instrumentação, facilitando os processos de irrigação e obturação dos canais radiculares, e ainda, possibilita avaliar a necessidade do refinamento apical com limas manuais na ausência de instrumentos rotatórios compatíveis com a anatomia apical, contribuindo para o sucesso da terapia endodôntica.

\section{Conclusão}

Concluiu-se que o diâmetro foraminal pode variar conforme o tipo de dente/canal, devido as características anatômicas de cada dente. A faixa etária do paciente e o diagnóstico pulpar e perirradicular do elemento dental quando analisados simultaneamente, também podem influenciar no diâmetro foraminal.

\section{Referências}

Abarca, J., Zaror, C., Monardes, H., Hermosilla, V., Muñoz, C., \& Cantin, M. (2014). Morphology of the physiological apical foramen in maxillary and mandibular first molars. International journal of morphology= Revista internacional de morfología, $32(2), 671$.

Campello, A. F., Marceliano-Alves, M. F., Siqueira Jr, J. F., Marques, F. V., Guedes, F. R., Lopes, R. T., ... \& Alves, F. R. (2019). Determination of the initial apical canal diameter by the first file to bind or cone-beam computed tomographic measurements using micro-computed tomography as the gold standard: an ex vivo study in human cadavers. Journal of endodontics, 45(5), 619-622.

Contreras, M. A. L., Zinman, E. H., \& Kaplan, S. K. (2001). Comparison of the first file that fits at the apex, before and after early flaring. Journal of Endodontics, 27(2), 113-116.

Demiriz, L., Bodrumlu, E. H., \& Icen, M. (2018). Evaluation of root canal morphology of human primary mandibular second molars by using cone beam computed tomography. Nigerian journal of clinical practice, 21(4).

Gani, O., \& Visvisian, C. (1999). Apical canal diameter in the first upper molar at various ages. Journal of Endodontics, $25(10), 689-691$.

Green, E. N. (1958). Microscopic investigation of root canal diameters. The Journal of the American Dental Association, 57(5), 636-644.

Jou, Y. T., Karabucak, B., Levin, J., \& Liu, D. (2004). Endodontic working width: current concepts and techniques. Dental Clinics, $48(1)$, $323-335$.

Kerekes, K., \& Tronstad, L. (1977). Morphometric observations on the root canals of human molars. Journal of Endodontics, 3(3), $114-118$.

Kfir, A., Rosenberg, E., \& Fuss, Z. (2006). Comparison in vivo of the first tapered and nontapered instruments that bind at the apical constriction. Oral Surgery, Oral Medicine, Oral Pathology, Oral Radiology, and Endodontology, 102(3), 395-398.

Kuttler, Y. (1955). Microscopic investigation of root apexes. The Journal of the American Dental Association, $50(5), 544-552$.

Levorato, G. L., Pereira, E. R., Carnevalli, B., \& Franco de Carvalho, E. M. O. (2011). Avaliação da forma e dos diâmetros cervical, médio e apical dos canais principais e dos forames apicais dos molares superiores: parte II. Rev. odontol. UNESP (Online).

Lopes, H. P., Siqueira Júnior, J. F., Elias, C. N., \& Vieira, M. V. B. (2015). Preparo químico-mecânico dos canais radiculares. In: Lopes HP, Siqueira Júnior JF. Endodontia: biologia e técnica. 4a ed. Rio de Janeiro: Guanabara Koogan; 355.

Madeira, M. C., \& Rizzolo, R. J. T. (2007). Cavidade pulpar. In: Madeira MC. Anatomia do dente. (5a ed.), Sarvier. 101

Marroquín, B. B., El-Sayed, M. A., \& Willershausen-Zönnchen, B. (2004). Morphology of the physiological foramen: I. Maxillary and mandibular molars. Journal of Endodontics, 30(5), 321-328.

Morfis, A., Sylaras, S. N., Georgopoulou, M., Kernani, M., \& Prountzos, F. (1994). Study of the apices of human permanent tee th with the use of a scanning electron microscope. Oral surgery, oral medicine, oral pathology, 77(2), 172-176.

Nitzan, D. W., Michaeli, Y., Weinreb, M., \& Azaz, B. (1986). The effect of aging on tooth morphology: a study on impacted tee th. Oral Surgery, Oral Medicine, Oral Pathology, 61(1), 54-60.

Pecora, J. D., Capelli, A., Guerisoli, D. M. Z., Spanó, J. C. E., \& Estrela, C. (2005). Influence of cervical preflaring on apical file size determination. International Endodontic Journal, 38(7), 430-435.

Peters, A. O., \& Peters, C. I. (2011). Limpeza e modelagem do sistema de canais radiculares. In: Hargreaves KM, Cohen S. Caminhos da Polpa. (10a ed.), Elsevier, 270-271

Ricucci, D., Pascon, E. A., \& Siqueira, J. F. (2019). The Complexity of the Apical Anatomy. In The Root Canal Anatomy in Permanent Dentition (pp. 241-254). Springer, Cham.

Schilder, H. (1974). Cleaning and shaping the root canal. Dent Clin North Am, 18(2):269-296.

Singh, S. K., Kanaparthy, A., Kanaparthy, R., Pillai, A., \& Sandhu, G. (2013). Geriatric endodontic. Journal of Orofacial Research, $191-196$.

Tan, B. T., \& Messer, H. H. (2002). The effect of instrument type and preflaring on apical file size determination. International Endodontic Journal, 35(9), 752758. 
Research, Society and Development, v. 10, n. 6, e5810615428, 2021

(CC BY 4.0) | ISSN 2525-3409 | DOI: http://dx.doi.org/10.33448/rsd-v10i6.15428

Versiani, M. A., Leoni, G. B., Pécora, J. D., \& Sousa Neto, M. D. (2015). Anatomia interna. In: Lopes, H. P., Siqueira Júnior, J. F. Endodontia: biologia e técnica. 4a ed. Rio de Janeiro: Guanabara Koogan, p. 206-211.

Vier, F. V., Tochetto, F. F., Orlandin, L. I., Xavier, L. L., Michelon, S., \& Barletta, F. B. (2004). Avaliação in vitro do diâmetro anatômico de canais radiculares de molares humanos, segundo a influência da idade.

Wolf, T. G., Paqué, F., Woop, A. C., Willershausen, B., \& Briseño-Marroquín, B. (2017). Root canal morphology and configuration of 123 maxillary second molars by means of micro-CT. International journal of oral science, 9(1), 33-37.

Wu, M. K., Barkis, D., Roris, A., \& Wesselink, P. R. (2002). Does the first file to bind correspond to the diameter of the canal in the apical region? International Endodontic Journal, 35(3), 264-267.

Wu, M. K., R'oris, A., Barkis, D., \& Wesselink, P. R. (2000). Prevalence and extent of long oval canals in the apical third. Oral Surgery, Oral Medicine, Oral Pathology, Oral Radiology, and Endodontology, 89(6), 739-743. 